\title{
A Mindfulness Program to Improve Resident Physicians' Personal and Work-Related Well-being: a Feasibility Study
}

\author{
Johannes C. Fendel ${ }^{1}$ (1) $\cdot$ Vanessa M. Aeschbach ${ }^{2} \cdot$ Anja S. Göritz $^{1} \cdot$ Stefan Schmidt $^{2}$
}

Published online: 16 April 2020

(C) The Author(s) 2020

\begin{abstract}
Objectives Many resident physicians suffer from mental distress. This endangers the individual physician as well as the quality of patient care. This study tested the feasibility of a novel 8-week mindfulness program tailored to improve resident physicians' personal and work-related well-being.

Methods Participants were nine resident physicians from a major hospital in southern Germany. Feasibility was assessed in seven domains (demand, acceptability, implementation, practicality, adaptation, integration, and preliminary effectiveness), using selfreports, attrition, attendance, and daily home practice. Using a pre-post within-subjects design, changes were assessed in hair cortisol as a biomarker of stress as well as in self-reported personal and work-related well-being.

Results The program is feasible in all domains. Participants positively rated the program regarding satisfaction, helpfulness, usage, and personal and professional benefit. All participants indicated to recommend the program. There was no study attrition, high attendance, and an average daily home practice of $13.5 \mathrm{~min}$. A medium reduction was observed with hair cortisol secretion $(d=0.64)$. In terms of personal well-being, medium-to-large improvements were observed with perceived stress $(d=0.58)$, mental health $(d=0.77)$, self-attributed mindfulness $(d=0.92)$, and self-compassion $(d=1.21)$. In terms of work-related wellbeing, small-to-medium improvements were observed with self-reported job strain $(d=0.58)$, effort-reward imbalance $(d=0.36)$, work-related burnout $(d=0.32)$, thriving at work $(d=0.24)$, and physician empathy $(d=0.21)$.

Conclusions This study provides evidence that a tailored mindfulness program is feasible and may be effective in reducing hair cortisol secretion and improving resident physicians' personal and work-related well-being. These promising findings warrant further investigation within a randomized controlled trial.
\end{abstract}

Keywords Mindfulness $\cdot$ Resident physician $\cdot$ Feasibility $\cdot$ Hair cortisol $\cdot$ Well-being

Medical residency is a stressful and demanding period in a physicians' professional life. A multitude of work stressors, including high workload, excessive time demands, scarcity of supervisory support, and restricted autonomy, endanger resident physicians' well-being (Prins et al. 2007). Through growing economic pressure, performance orientation, and high administrative load, resident physicians often become

Johannes C. Fendel

johannes.fendel@psychologie.uni-freiburg.de

1 Department of Occupational and Consumer Psychology, Institute of Psychology, University of Freiburg, Engelbergerstraße 41, 79085 Freiburg, Germany

2 Department for Psychosomatic Medicine and Psychotherapy, Medical Center - University of Freiburg, Faculty of Medicine, University of Freiburg, Freiburg, Germany disillusioned with modern medical practice (Beerheide 2017). Consequently, resident physicians more suffer from burnout and depression in comparison with the general population (Dyrbye et al. 2014) and are less satisfied with their life (Tyssen et al. 2009). The high levels of burnout during residency have detrimental personal consequences such as substance abuse (Lebensohn et al. 2013) and suicidal thoughts (van der Heijden et al. 2008). Furthermore, when suffering from burnout resident physicians commit more medical errors (Baer et al. 2017; Prins et al. 2009), express lower empathy (Ferreira et al. 2020; Park et al. 2016), and adhere less to practice and safety standards (de Oliveira et al. 2013).

A promising approach to improve resident physicians' well-being is the practice of mindfulness. Mindfulness is commonly described as moment-to-moment awareness by paying attention to the present moment in a non-reactive, non-judgmental, and openhearted manner (Kabat-Zinn 2005, p. 108). It 
can be cultivated through a range of formal and informal practices and is often taught in mindfulness-based programs (MBPs). MBPs have been shown to be effective in reducing physicians' stress, burnout, depression, and anxiety (Fortney et al. 2013) and seem to improve quality of care: After participation in an MBP, physicians reported increases in empathy (Krasner et al. 2009) and dedication to work (Verweij et al. 2016).

However, research on the effectiveness of MBPs for resident physicians, as a group that is particularly burdened, is scarce. First results are promising, but somewhat heterogeneous. Some studies reported decreases in stress or burnout (Ireland et al. 2017; Lases et al. 2016), whereas other studies failed to find such associations (Goldhagen et al. 2015; Verweij et al. 2018). The participants of an MBP for surgical resident physicians suggested that the program should have had a stronger focus on their daily medical practice, and the authors conclude that MBPs for resident physicians are probably most effective when tailored to meet this group's particular needs (Lases et al. 2016). We share this opinion and believe that an MBP for resident physicians should take into account the complex interplay of the specific work stressors, personality traits, and maladaptive attitudes that account for resident physicians' distress. Perfectionism, workaholism, and neglect of self-care are more prevalent among physicians and are associated with adverse health outcomes (Wallace et al. 2009). Moreover, the culture of medical training leads resident physicians to adopt self-critical and excessively selfsacrificing attitudes and to prioritize professional accomplishments over personal needs (Irving et al. 2009). Thus, any MBP for this particular target group should be primarily oriented towards the improvement of well-being and basic attitudinal qualities rather than symptom alleviation and performance increase. Otherwise, resident physicians may run the risk of misconceiving a mindfulness program as yet another means of optimizing their performance.

In terms of evaluation, there has been a call for the inclusion of biomarkers when investigating the effect of MBPs (Irving et al. 2009). Cortisol is a steroid hormone released in response to stressful events. It has been used in MBP research, as stemming from saliva, blood, or urine (Sanada et al. 2016). However, these assays provide a measurement at a single point in time and only reflect acute (blood, saliva) or shortterm fluctuations (urine) in cortisol secretion. In contrast, assessing cortisol levels from hair samples provides a promising indicator of longer-term cortisol secretion and offers a unique possibility to retrospectively monitor chronic stress (Stalder and Kirschbaum 2012). Furthermore, when evaluating MBPs, research linking mindfulness to empathy, flourishing, or self-compassion has encouraged to include salutogenetic outcomes (Irving et al. 2009).

The following study tests the feasibility of a novel mindfulness program tailored to the particular needs and circumstances of resident physicians, in order to improve their personal and work-related well-being. The study provides tentative effect size estimations and determines whether conducting a larger randomized controlled trial is warranted. Beyond self-reports on symptoms of stress, the present study assesses cortisol in human hair as a biomarker of stress as well as salutogenetic outcomes.

\section{Methods}

\section{Participants}

We invited all 150 resident physicians of a major hospital in southern Germany in November 2017 to voluntarily participate in an 8-week mindfulness program. Physicians from all stages and disciplines of residency were eligible for inclusion as well as physicians who finished residency in the year of baseline assessment. Nine resident physicians (five women and four men; mean age $=33.2 ; S D=4.06$ ) participated. All physicians reported to have had no prior meditation experience. Average working hours were $46.0 \mathrm{~h}$ per week $(S D=$ 8.29), and all participants reported to be in a stable romantic relationship.

\section{Procedure}

Within an observational study design, 1 week before and after the mindfulness program, participants provided hair samples and completed online self-report questionnaires. At the preprogram testing session, participants gave written informed consent. The Ethics Board of the Freiburg University Medical Center approved the study.

\section{Program}

We based the program on the well-validated Mindfulnessbased stress reduction program (MBSR; Kabat-Zinn 1990) and tailored it to resident physicians' particular needs and circumstances. To this end, we conducted an a priori needs assessment, consisting of an extensive literature search as well as in-depth interviews with resident and practicing physicians, and accomplished the following programmatic modifications. First, we incorporated themes of relevance in resident physicians' daily practice that go beyond the standard MBSR program (i.e., typical resident physician stressors like high responsibility or fear of committing errors). An outline of the session themes and a summary of contents are in Table 1. Second, in order to address resident physicians' well-being, we oriented the program towards promoting the experience of Muße. The term Muße is well-known in German but cannot be directly translated into English. It can be defined as a state of being in which individuals feel liberated of pressures of all 
Table 1 Session outline

\begin{tabular}{|c|c|c|}
\hline Week & Session theme & Summary of contents \\
\hline 1 & Mindfulness and Muße & $\begin{array}{l}\text { Exploring resident physicians' needs and expectations; introducing mindfulness and Muße as modes } \\
\text { of being, as contrasted to modes of doing and performing; exploring ways to experience Muße in } \\
\text { medical practice. }\end{array}$ \\
\hline 2 & $\begin{array}{l}\text { Dealing with barriers and subjective } \\
\text { perception of time }\end{array}$ & $\begin{array}{l}\text { Discussing ways to deal with barriers to mindful practice; exploring mindful anchors in daily routine } \\
\text { (e.g., mindful walking along hospital corridors; mindful stop before entering a patient's room; } \\
\text { mindful hand disinfection); introducing mindfulness to experience slower passage of time and to } \\
\text { mitigate the feeling of time pressure. }\end{array}$ \\
\hline 3 & Dis-identification & $\begin{array}{l}\text { Coping with painful emotions, thoughts, and physical sensations; raise awareness of the process of } \\
\text { constructing reality through one's experiences; connecting with the inner observer and exploring } \\
\text { dis-identification to learn to non-identify with thoughts and feelings and to reduce reactivity towards } \\
\text { them. }\end{array}$ \\
\hline 4 & Stress & $\begin{array}{l}\text { Discussing resident physicians' specific stressors; psychoeducation on physiological and } \\
\text { psychological processes of stress; exploring how to cope with stress using mindfulness. }\end{array}$ \\
\hline \multirow[t]{2}{*}{5} & Acceptance & $\begin{array}{l}\text { Learning acceptance of oneself as well as the given reality of experiences, events, and working } \\
\text { conditions. Exploring the difference between acceptance and resignation/fatalism and the impor- } \\
\text { tance of acceptance for self-care. }\end{array}$ \\
\hline & Day of mindfulness & $\begin{array}{l}\text { All-day silent retreat. Practicing mindfulness intensively; reinforcing mindfulness as a reliable tool in } \\
\text { both everyday life and daily medical practice. }\end{array}$ \\
\hline 6 & Mindfulness in patient contact & $\begin{array}{l}\text { Using mindfulness in therapeutic interactions; building up a compassionate communication } \\
\text { atmosphere with patients, even in moments of time pressure. Learning to listen mindfully and } \\
\text { exploring the benefits of letting patients complete their agenda of concerns. }\end{array}$ \\
\hline 7 & Self-care & $\begin{array}{l}\text { Discussing why self-care is especially relevant to resident physicians and its connection to quality of } \\
\text { care; exploring ways to take care of oneself in daily routine. }\end{array}$ \\
\hline 8 & $\begin{array}{l}\text { Enhancing meaning in work and } \\
\text { mindfulness as part of life }\end{array}$ & $\begin{array}{l}\text { Exploring what is meaningful in professional life and how meaning may be enhanced in health care. } \\
\text { Reinforcing mindfulness as part of everyday life and daily medical practice. }\end{array}$ \\
\hline
\end{tabular}

kind, especially time pressure and the pressure to perform (Gouda et al. 2016). In this sense, Muße serves as an antidote to performance and self-optimization attitudes, emphasizes the self-care and self-compassion components of mindfulness, and is associated with feelings of enjoyment, fulfillment, serenity, and freedom. Third, we facilitated transfer by including practical exercises focusing on resident physicians' daily practice and encouraged participants to practice mindfulness during everyday routine (e.g., feeling one's feet while walking down the hospital corridor, feeling one's hands during hand disinfection). Fourth, we provided participants with detailed background information underpinning course content with explanatory models and scientific evidence.

The mindfulness program consisted of eight weekly 135 min sessions plus a full-day 6-h silent retreat. Every session comprised the following five components: (1) theoretical input (20 min: see Table 1), (2) formal mindfulness practices (45 $\mathrm{min}$ ), (3) group inquiry (40 $\mathrm{min})$, (4) transfer to daily routine $(25 \mathrm{~min})$, and $(5)$ home assignments $(5 \mathrm{~min})$. In line with previous studies, study completion was defined as having attended four or more sessions (Verweij et al. 2018).

\section{Instructors}

Two experienced meditators who are trained medical psychiatrists, psychotherapists, and highly experienced mindfulness instructors delivered the program. They are certified by the German MBSR-MBCT teacher association.

\section{Measures}

\section{Feasibility}

We assessed the feasibility of the program on seven dimensions (Bowen et al. 2009): Demand was derived from the alarming prevalence of mental distress among resident physicians in the literature (see above) and from interviews we conducted in the design phase of the program. Acceptability was operationalized as overall program satisfaction, perceived helpfulness of session components, personal and professional benefit, and willingness to recommend the program to peers. Implementation was operationalized as the extent to which the program could be fully delivered. It was evaluated by the number of canceled, truncated, or postponed sessions as well as participants' satisfaction with the general conditions in terms of program organization, time requirements, premises, and meditation materials. Practicality was evaluated by program attendance, duration of daily home practice, and monetary costs. Adaptation was operationalized as modifications made to the classical MBSR program to tailor the program to resident physicians' particular needs and circumstances. Integration was operationalized as the extent to which 
participants used the learned techniques in everyday life and how they plan to do so in the future. Preliminary effectiveness was operationalized as effect size estimations of changes in hair cortisol and self-reports of personal and work-related well-being.

\section{Hair Cortisol}

At the pre- and post-program testing session, we cut two hair strands of a diameter of $3 \mathrm{~mm}$ as close as possible to the scalp from a posterior vertex position. Cortisol concentration from the 1-cm hair segment closest to the scalp was determined via the immunoassay method (Staufenbiel et al. 2013). As hair grows on average $1 \mathrm{~cm}$ every 4 weeks, the analyzed segments reflect the cumulative cortisol secretion over the period of 4 weeks prior to hair sampling (Kirschbaum et al. 2009). Exclusion criteria for hair analyses are chemical hair treatments such as dyeing or tinting (Sauvé et al. 2007), baldness, and pregnancy (Dettenborn et al. 2012).

\section{Self-reports on Personal Well-being}

We used an online survey platform (Unipark EFS Survey) and included German versions of validated self-report scales; all Cronbach's alphas stem from the current sample. The Perceived Stress Scale (PSS; Klein et al. 2016; $\alpha=0.80$ ) measures the frequency of stress-related feelings and thoughts during the past month. The General Health Questionnaire (GHQ-12; Romppel et al. 2013; $\alpha=0.93$ ) measures mental health and identifies psychological and minor psychiatric problems. Personal burnout is a subscale of the Copenhagen Burnout Inventory (CBI; Hanebuth et al. 2012; $\alpha=0.77$ ). The short version of the Freiburg Mindfulness Inventory (FMI-14; Walach et al. 2006; $\alpha=0.82$ ) measures self-attributed mindfulness on the two subscales presence and acceptance. The short version of the Self-Compassion Scale (SCS-SF; Hupfeld and Ruffieux 2011; $\alpha=0.65$ ) measures self-compassion. The Subjective Time Questionnaire (STQ; Wittmann et al. 2015) measures the subjective passage of time on three subscales: the feeling of time pressure $(\alpha=$ $0.71)$, the feeling of time expansion $(\alpha=0.58)$, and the feeling of routines in life $(\alpha=0.89)$.

\section{Self-reports on Work-Related Well-being}

Work-related burnout $(\alpha=0.82)$ and client-related burnout $(\alpha=0.84)$ are subscales of the above described CBI. The Irritation Scale (IS; Mohr et al. 2005; $\alpha=0.87$ ) measures perceived job strain on the two subscales cognitive irritation $(\alpha=0.86)$ and emotional irritation $(\alpha=0.88)$. The EffortReward Imbalance questionnaire (ERI-16; Siegrist et al. 2009) identifies adverse health effects of stressful experience at work on the three subscales effort $(\alpha=0.71)$, reward ( $\alpha=$
$0.57)$, and overcommitment $(\alpha=0.80)$. The core assumption of the model is social reciprocity in occupational life, meaning that workers expect rewards (e.g., money, esteem) for their work effort (e.g., job demands; Siegrist 1996). Imbalance is computed by a ratio between effort and reward, corrected by the number of items of each subscale. Values $>1$ indicate increased effort not met by the rewards received in return (Siegrist et al. 2004). The model is expanded by overcommitment, a trait component that acts as a personal risk factor that can maintain and intensify any effort-reward imbalance (Niedhammer et al. 2004). The Thriving at Work Scale (Hildenbrand et al. 2018; $\alpha=0.93$ ) measures thriving, defined as "the psychological state in which individuals experience both a sense of vitality and a sense of learning at work" (Spreitzer et al. 2005, p. 538). The Faces Scale measures the current level of job satisfaction with one item (Kunin 1955). We measured physician empathy using six items of the Jefferson Scale of Physician Empathy (JSPE; Fischer et al. as cited in Doering et al. 2010; $\alpha=0.94$ ).

\section{Data Analyses}

We used SPSS version 24.0 (IBM Corp., Armonk, NY, USA) to conduct dependent sample $t$ tests and, whenever pre-post differences where not normally distributed, non-parametric Wilcoxon's signed-rank test (both two-tailed). However, due to the small sample and the feasibility character of this study, inference statistics should be interpreted with caution. We interpreted standardized effect size estimates of 0.2 as small, 0.50 as medium, and 0.80 or greater as large (Cohen 1988).

\section{Results}

Demand The interviews during the needs assessment confirmed that there is a great deal of dissatisfaction and disillusion with medical practice, a strong need to improve resident physicians' well-being and a longing for ways of dealing with internal and external stressors.

Acceptability The mean ratings for overall satisfaction with program and trainers as well as the ratings for personal and professional benefit were very high. The session components were perceived as helpful and all participants highly recommend the program (Table 2).

Implementation No session was canceled and the planned curriculum was fully delivered. Satisfaction with the general conditions was high and ranged between 4.11 and 4.89 (Table 2).

Practicality All participants completed the program and attended at least five out of nine sessions $(M=6.44$, 
$S D=1.33)$. They indicated to have spent on average $13.49 \min (S D=16.02$; median $=10)$ on home practice each day during the eight program weeks. The monetary costs consisted of the payments for supervision in the design phase (3.000€), program delivery (4.000€), and meditation materials $(1.400 €)$.

Adaptation We based our program on the well-validated MBSR program and tailored it to resident physicians' particular needs and circumstances. Modifications included the thematic focus (Table 1), the orientation towards Muße, the facilitation of transfer to daily practice, and the explanatory scientific foundation. The notes of the instructors taken after each session were discussed with the authors of this study, and changes for future applications of the program were integrated into the curriculum.

Integration Participants indicated to use the learned techniques in everyday life $(M=3.78)$ and plan to do so in the future $(M=4.11$; Table 2).

Preliminary Effectiveness Table 3 displays pre- and postprogram mean scores, standard deviations, and effect sizes for hair cortisol secretion and self-reported personal and work-related well-being. Scores across all measures except those of the Subjective Time Questionnaire and clientrelated burnout trended in the expected direction.

\section{Discussion}

The present study gauged the feasibility of an 8-week mindfulness program tailored towards improving the well-being of resident physicians. Beyond self-reports on symptoms of stress, this study includes hair cortisol as an objective biomarker of stress as well as salutogenetic outcomes. The results provide evidence on the feasibility of the program and give hints to its potential effectiveness in reducing cortisol secretion and in improving self-reported personal and work-related well-being.

The results on all eight dimensions demonstrate a high feasibility and a successful tailoring of the program. The homogeneity of the group consisting exclusively of resident physicians may have helped participants in sharing, recognizing, and normalizing personal stress symptoms and may have encouraged them to open up and learn from one another (Beckman et al. 2012). With regard to preliminary effectiveness, except the subscales of the STQ, scores across all measures trended in the expected direction, and many effect sizes are in the range of clinical significance.

Table 2 Resident physicians' program evaluation scores

\begin{tabular}{|c|c|c|}
\hline Evaluation domains and items & Mean (SD) & Min-max \\
\hline \multicolumn{3}{|l|}{ Overall satisfaction ( 1 = very dissatisfied; 5 = very satisfied $)$} \\
\hline How satisfied were you with the program? & $4.89(0.33)$ & $4-5$ \\
\hline How satisfied were you with the trainers? & $5.00(0.00)$ & $5-5$ \\
\hline \multicolumn{3}{|l|}{ Helpfulness of session components ( 1 = not helpful; 5 = very helpful $)$} \\
\hline How helpful was the theoretical input? & $4.00(0.71)$ & $3-5$ \\
\hline How helpful were the mindfulness meditations? & $4.44(0.73)$ & $4-5$ \\
\hline How helpful was the inquiry among colleagues? & $4.44(0.53)$ & $4-5$ \\
\hline How helpful was the transfer to daily practice? & $3.89(0.93)$ & $2-5$ \\
\hline How helpful were the audio guides for home assignments? & $4.44(0.53)$ & $4-5$ \\
\hline \multicolumn{3}{|l|}{ Benefit ( $1=$ no benefit; 5 = great benefit $)$} \\
\hline How do you rate your professional benefit of the program? & $4.78(0.44)$ & $4-5$ \\
\hline How do you rate your personal benefit of the program? & $4.78(0.44)$ & $4-5$ \\
\hline \multicolumn{3}{|l|}{ Recommendation $(1=$ no; $5=$ yes $)$} \\
\hline Would you recommend the program? & $4.89(0.33)$ & $4-5$ \\
\hline \multicolumn{3}{|l|}{ Satisfaction with general conditions ( 1 = very dissatisfied; 5 = very satisfied $)$} \\
\hline How satisfied were you with the program organization? & $4.89(0.33)$ & $4-5$ \\
\hline How satisfied were you with time requirements? & $4.11(0.60)$ & $3-5$ \\
\hline How satisfied were you with the premises? & $4.33(0.50)$ & $4-5$ \\
\hline How satisfied were you with the meditation materials (pillows, mats, blankets)? & $4.78(0.44)$ & $4-5$ \\
\hline \multicolumn{3}{|l|}{ Integration $(1=$ never; 5 = every day $)$} \\
\hline How often do you use the learned techniques in everyday life? & $3.78(0.67)$ & $3-5$ \\
\hline Do you plan to use the learned techniques in the future? & $4.11(0.60)$ & $3-5$ \\
\hline
\end{tabular}


Table 3 Pre- and post-program scores for self-reports and hair cortisol secretion

\begin{tabular}{|c|c|c|c|c|c|c|}
\hline \multirow[t]{2}{*}{ Measure $(n=9)$} & \multicolumn{2}{|c|}{ Pre-test } & \multicolumn{2}{|c|}{ Post-test } & \multirow[t]{2}{*}{$p^{a}$} & \multirow[t]{2}{*}{ Effect size $\left(\mathrm{SES}^{\mathrm{b}}\right)$} \\
\hline & M & SD & M & $\mathrm{SD}$ & & \\
\hline Hair cortisol ${ }^{\mathrm{c}}$ & 6.35 & 0.85 & 5.80 & 1.18 & .16 & 0.64 \\
\hline \multicolumn{7}{|l|}{ Personal well-being } \\
\hline PSS & 21.33 & 5.00 & 18.44 & 5.77 & .11 & 0.58 \\
\hline GHQ-12 & 14.33 & 6.46 & 9.33 & 3.54 & .10 & 0.77 \\
\hline CBI-Personal & 3.35 & 0.58 & 3.24 & 0.58 & .36 & 0.19 \\
\hline FMI-14 & 30.67 & 6.02 & 36.22 & 4.24 & .02 & 0.92 \\
\hline SCS-D short form & 2.57 & 0.50 & 3.18 & 0.63 & .001 & 1.21 \\
\hline \multicolumn{7}{|l|}{ STQ } \\
\hline Time pressure & 3.57 & 0.68 & 3.76 & 0.62 & .34 & -0.29 \\
\hline Time expansion & 1.47 & 0.57 & 1.27 & 0.37 & .18 & -0.35 \\
\hline Routines & 3.06 & 1.18 & 3.06 & 0.77 & $.94^{\mathrm{d}}$ & 0.00 \\
\hline \multicolumn{7}{|c|}{ Work-related well-being } \\
\hline CBI work-related & 3.00 & 0.55 & 2.83 & 0.54 & .11 & 0.32 \\
\hline CBI client-related & 2.67 & 0.75 & 2.65 & 0.64 & .93 & 0.02 \\
\hline \multicolumn{7}{|l|}{ IS } \\
\hline Total & 4.15 & 1.29 & 3.40 & 1.27 & .02 & 0.58 \\
\hline Cognitive & 4.22 & 1.58 & 3.33 & 1.72 & .02 & 0.56 \\
\hline Emotional & 4.11 & 1.47 & 3.44 & 1.28 & .046 & 0.45 \\
\hline \multicolumn{7}{|l|}{ ERI-16 } \\
\hline Ratio & 1.56 & 0.39 & 1.42 & 0.38 & .045 & 0.36 \\
\hline Effort & 11.00 & 1.32 & 10.56 & 1.13 & $.36^{\mathrm{d}}$ & 0.34 \\
\hline Overcommitment & 15.33 & 4.00 & 14.56 & 3.40 & .35 & 0.19 \\
\hline Reward & 16.89 & 2.67 & 17.89 & 2.52 & $.03^{\mathrm{d}}$ & 0.37 \\
\hline Thriving at work & 3.79 & 0.90 & 4.00 & 0.61 & .43 & 0.24 \\
\hline Job satisfaction & 4.56 & 1.13 & 4.67 & 1.50 & $.56^{\mathrm{d}}$ & 0.10 \\
\hline JSPE & 33.33 & 7.89 & 35.00 & 6.86 & .17 & 0.21 \\
\hline
\end{tabular}

PSS, Perceived Stress Scale; GHQ-12, General Health Questionnaire; CBI, Copenhagen Burnout Inventory; FMI-14, Freiburg Mindfulness Inventory; $S C S$-D short form, Self-Compassion Scale; STQ, Subjective Time Questionnaire; IS, Irritation Scale; ERI-16, Effort-Reward Imbalance; JSPE, Jefferson Scale of Physician Empathy

${ }^{\text {a }}$ Paired $t$ test (two-tailed)

${ }^{\mathrm{b}}$ SES standardized effect size. Changes in the expected direction are positively coded; changes in the unexpected direction are negatively coded

${ }^{\mathrm{c}}$ Due to dyeing and pregnancy, two participants were excluded from hair cortisol analyses $(n=7)$

${ }^{\mathrm{d}}$ Wilcoxon's signed-rank test (two-tailed), as pre-post difference was not normally distributed

Concerning personal well-being, our findings on hair cortisol are in line with Goldberg et al. (2014) who described a medium-sized reduction in hair cortisol secretion after a 7week mindfulness training for smoking cessation. Furthermore, the self-reports of stress and mental health indicated a medium-sized improvement. This is comparable with that of Lases et al. (2016) who described a medium reduction in stress after a 12 -week MBP for surgical resident physicians. Comparable with previous studies, we did not find substantial improvements in personal burnout (Goldhagen et al. 2015; Verweij et al. 2018). Importantly, we found large increases in self-compassion and selfattributed mindfulness. This might explain the observed improvements in personal well-being (Gu et al. 2015), as resident physicians after completing the program may have paid more attention to their needs and improved their ability to regulate their thoughts and emotions (Tang et al. 2015). With regard to the subjective passage of time, we found small decreases in the feeling of expansion of time, small increases in the feeling of time pressure, and no change in the feeling of routines. This finding, if not a chance finding, suggests that after the program, participants felt somewhat more time pressure and less time dilation. This is unlike what we expected, as it has been proposed that subjective time slows down in mediation practice (Wittmann and Schmidt 2014). Therefore, there is a need for further investigations 
with greater sample sizes to examine the influence of MBPs on resident physicians' subjective passage of time.

Concerning work-related well-being, we found small-tomedium improvements in job strain, thriving at work, physician empathy, and the effort-reward ratio. These findings may be interpreted in the way that the practice of mindfulness may have helped the resident physicians to be absorbed less by the demanding aspects of medical practice. Instead, they may have learned to recognize and connect with those aspects most meaningful to them as well as to experience the rewards that come from restoring health and relieving suffering (Epstein 1999; Shanafelt 2009). We found small reductions in workrelated burnout but hardly any improvements in client-related burnout. These results are consistent with meta-analytic findings for studies with healthy individuals (Khoury et al. 2015), indicating that MBPs only have small effects on burnout and might be beneficial in more general terms of well-being. In line with Lases et al. (2016), we did not find substantial changes in job satisfaction. The observed improvements in different facets of work-related well-being point out the program's potential for positive outcomes to translate into improved patient care.

\section{Limitations and Future Research}

The small number of participants, the lack of a randomized control group, the absence of follow-up data, and the selfselection of participation limit the generalizability of the results and preclude causal inferences about the effectiveness of the tailored mindfulness program at this time. However, this trial was not designed to assess effectiveness of the program, but its goal was to test its feasibility and the implementation of assessment methods as well as to give tentative effect-size estimates in order to conduct an empirically informed power analysis for a larger controlled trial. Moreover, MBPs are believed most effective if individuals choose to engage (Walach et al. 2014). Therefore, self-selection is a standard and preferred practice for this type of program (Burton et al. 2017). Furthermore, since participation in the program is time-intense, resident physicians may be dissuaded from choosing to participate. Nevertheless, as mindfulness once learned is not tied to times and places, it can easily and flexibly be implemented into daily life (Ireland et al. 2017). In addition to effects of program participation on the level of individual program participants, future studies should evaluate transfer effects onto the participant's environment, for example, by including reports by patients and colleagues.

Acknowledgments We thank the Collaborative Research Center 1015 Otium. Boundaries, Chronotopes, Practices for supporting the project. Special thanks go to Susanne Bregulla-Kuhn and Klaus Kuhn for their creativity and exceptional guidance during the design process and for their outstanding commitment during the implementation of the program and the subsequent final adaptations. Without their support, the conception of the program would not have been possible. We also thank Susanne Rausch and Elina Kraemer for their assistance.

Authors' Contributions All authors contributed to the design of the program and the study. SS and ASG developed the initial concept, secured funding and supervised all stages of the research. JCF and VMA implemented the trial, handled the participants and recorded all data. JCF conducted the data analyses and wrote the manuscript. VMA, ASG and SS made valuable revisions to the manuscript.

Funding Information Open Access funding provided by Projekt DEAL. This study was funded by the Deutsche Forschungsgemeinschaft (DFG, German Research Foundation - Project number 197396619 SFB 1015).

Data Availability All data will be made available upon request from the corresponding author.

\section{Compliance with Ethical Standards}

The study was approved by the Ethics Board of the Medical Center University of Freiburg. All procedures were in accordance with the ethical standards laid down in the 1964 Helsinki Declaration and its later amendments.

Informed Consent Written informed consent was obtained from all individual participants included in the study.

Conflict of Interest The authors declare that they have no conflict of interest.

Open Access This article is licensed under a Creative Commons Attribution 4.0 International License, which permits use, sharing, adaptation, distribution and reproduction in any medium or format, as long as you give appropriate credit to the original author(s) and the source, provide a link to the Creative Commons licence, and indicate if changes were made. The images or other third party material in this article are included in the article's Creative Commons licence, unless indicated otherwise in a credit line to the material. If material is not included in the article's Creative Commons licence and your intended use is not permitted by statutory regulation or exceeds the permitted use, you will need to obtain permission directly from the copyright holder. To view a copy of this licence, visit http://creativecommons.org/licenses/by/4.0/.

\section{References}

Baer, T. E., Feraco, A. M., Sagalowsky, S. T., Williams, D., Litman, H. J., \& Vinci, R. J. (2017). Pediatric resident burnout and attitudes toward patients. Pediatrics, 139(3), e20162163. https://doi.org/10.1542/ peds.2016-2163.

Beckman, H. B., Wendland, M., Mooney, C., Krasner, M. S., Quill, T. E., Suchman, A. L., \& Epstein, R. M. (2012). The impact of a program in mindful communication on primary care physicians. Academic Medicine, 87(6), 815-819.

Beerheide, R. (2017). Deutsches Ärzteblatt: Junge Ärzte hadern mit Klinikalltag. Deutsches Ärzteblatt, 114(9), 399-400.

Bowen, D. J., Kreuter, M., Spring, B., Cofta-Woerpel, L., Linnan, L., Weiner, D., Bakken, S., Kaplan, C., Squiers, L., \& Fernandez, M. (2009). How we design feasibility studies. American Journal of Preventive Medicine, 36(5), 452-457. 
Burton, A., Burgess, C., Dean, S., Koutsopoulou, G. Z., \& Hugh-Jones, S. (2017). How effective are mindfulness-based interventions for reducing stress among healthcare professionals? A systematic review and meta-analysis. Stress and Health, 33(1), 3-13.

Cohen, J. (1988). Statistical power analysis for the behavioral sciences (2nd ed.). Hillsdale: Lawrence Erlbaum.

de Oliveira, G. S., Chang, R., Fitzgerald, P. C., Almeida, M. D., CastroAlves, L. S., Ahmad, S., \& McCarthy, R. J. (2013). The prevalence of burnout and depression and their association with adherence to safety and practice standards: a survey of United States anesthesiology trainees. Anesthesia \& Analgesia, 117(1), 182-193.

Dettenborn, L., Tietze, A., Kirschbaum, C., \& Stalder, T. (2012). The assessment of cortisol in human hair: associations with sociodemographic variables and potential confounders. Stress, 15(6), 578-588.

Doering, S., Schneider, G., Burgmer, M., Sensmeier, J., Schrewe, F. B., Friederichs, H., \& Heuft, G. (2010). Evaluation des Praktikums "Psychosomatik und Psychotherapie" mit standardisierten Patienten. Zeitschrift für Psychosomatische Medizin und Psychotherapie, 56(4), 385-398.

Dyrbye, L. N., West, C. P., Satele, D., Boone, S., Tan, L., Sloan, J., \& Shanafelt, T. D. (2014). Burnout among U.S. medical students, residents, and early career physicians relative to the general U.S. population. Academic Medicine, 89(3), 443-451.

Epstein, R. M. (1999). Mindful practice. JAMA, 282(9), 833-839.

Ferreira, S., Afonso, P., \& Ramos, M. d. R. (2020). Empathy and burnout: a multicentre comparative study between residents and specialists. Journal of Evaluation in Clinical Practice, 26(1), 216-222.

Fortney, L., Luchterhand, C., Zakletskaia, L., Zgierska, A., \& Rakel, D. (2013). Abbreviated mindfulness intervention for job satisfaction, quality of life, and compassion in primary care clinicians: a pilot study. The Annals of Family Medicine, 11(5), 412-420.

Goldberg, S. B., Manley, A. R., Smith, S. S., Greeson, J. M., Russell, E., Van Uum, S., Koren, G., \& Davis, J. M. (2014). Hair cortisol as a biomarker of stress in mindfulness training for smokers. The Journal of Alternative and Complementary Medicine, 20(8), 630-634.

Goldhagen, B., Kingsolver, K., Stinnett, S., \& Rosdahl, J. (2015). Stress and burnout in residents: impact of mindfulness-based resilience training. Advances in Medical Education and Practice, 525.

Gouda, S., Luong, M. T., Schmidt, S., \& Bauer, J. (2016). Students and teachers benefit from mindfulness-based stress reduction in a school-embedded pilot study. Frontiers in Psychology, 7(590), $1-18$.

Gu, J., Strauss, C., Bond, R., \& Cavanagh, K. (2015). How do mindfulness-based cognitive therapy and mindfulness-based stress reduction improve mental health and wellbeing? A systematic review and meta-analysis of meditation studies. Clinical Psychology Review, 37, 1-12.

Hanebuth, D., Aydin, D., \& Scherf, T. (2012). Burnout and related conditions in managers: a five-year longitudinal study. Psychology of Everyday Activity, 5(3), 17-50.

Hildenbrand, K., Sacramento, C. A., \& Binnewies, C. (2018). Transformational leadership and burnout: the role of thriving and followers' openness to experience. Journal of Occupational Health Psychology, 23(1), 31-43.

Hupfeld, J., \& Ruffieux, N. (2011). Validierung einer deutschen Version der Self-Compassion Scale (SCS-D). Zeitschrift für Klinische Psychologie und Psychotherapie, 40(2), 115-123.

Ireland, M. J., Clough, B., Gill, K., Langan, F., O’Connor, A., \& Spencer, L. (2017). A randomized controlled trial of mindfulness to reduce stress and burnout among intern medical practitioners. Medical Teacher, 39(4), 409-414.

Irving, J. A., Dobkin, P. L., \& Park, J. (2009). Cultivating mindfulness in health care professionals: a review of empirical studies of mindfulness-based stress reduction (MBSR). Complementary Therapies in Clinical Practice, 15(2), 61-66.
Kabat-Zinn, J. (1990). Full catastrophe living: the program of the stress reduction clinic at the University of Massachusetts Medical Center. New York: Delta.

Kabat-Zinn, J. (2005). Coming to our senses: healing ourselves and the world through mindfulness. London: Hachette UK.

Khoury, B., Sharma, M., Rush, S. E., \& Fournier, C. (2015). Mindfulness-based stress reduction for healthy individuals: a metaanalysis. Journal of Psychosomatic Research, 78(6), 519-528.

Kirschbaum, C., Tietze, A., Skoluda, N., \& Dettenborn, L. (2009). Hair as a retrospective calendar of cortisol production - increased cortisol incorporation into hair in the third trimester of pregnancy. Psychoneuroendocrinology, 34(1), 32-37.

Klein, E. M., Brähler, E., Dreier, M., Reinecke, L., Müller, K. W., Schmutzer, G., Wölfling, K., \& Beutel, M. E. (2016). The German version of the perceived stress scale - psychometric characteristics in a representative German community sample. BMC Psychiatry, 16(1), 159-168.

Krasner, M. S., Epstein, R. M., Beckman, H., Suchman, A. L., Chapman, B., Mooney, C. J., \& Quill, T. E. (2009). Association of an educational program in mindful communication with burnout, empathy, and attitudes among primary care physicians. JAMA, 302(12), 1284-1293.

Kunin, T. (1955). The construction of a new type of attitude measure. Personnel Psychology, 8(1), 65-77.

Lases, S. S., Lombarts, M. J. M. H., Slootweg, I. A., Arah, O. A., Pierik, E. G. J. M., \& Heineman, E. (2016). Evaluating mind fitness training and its potential effects on surgical residents' well-being: a mixed methods pilot study. World Journal of Surgery, 40(1), 29-37.

Lebensohn, P., Dodds, S., Benn, R., Brooks, A. J., \& Birch, M. (2013). Resident wellness behaviors. Family Medicine, 45(8), 541-549.

Mohr, G., Müller, A., \& Rigotti, T. (2005). Normwerte der Skala Irritation: zwei Dimensionen psychischer Beanspruchung. Diagnostica, 51(1), 12-20.

Niedhammer, I., Tek, M.-L., Starke, D., \& Siegrist, J. (2004). Effortreward imbalance model and self-reported health: cross-sectional and prospective findings from the GAZEL cohort. Social Science \& Medicine, 58(8), 1531-1541.

Park, C., Lee, Y. J., Hong, M., Jung, C.-H., Synn, Y., Kwack, Y.-S., Ryu, J.-S., Park, T. W., Lee, S. A., \& Bahn, G. H. (2016). A multicenter study investigating empathy and burnout characteristics in medical residents with various specialties. Journal of Korean Medical Science, 31(4), 590-597.

Prins, J. T., Gazendam-Donofrio, S. M., Tubben, B. J., van der Heijden, F. M. M. A., van de Wiel, H. B. M., \& Hoekstra-Weebers, J. E. H. M. (2007). Burnout in medical residents: a review. Medical Education, 41(8), 788-800.

Prins, J. T., van der Heijden, F. M. M. A., Hoekstra-Weebers, J. E. H. M., Bakker, A. B., van de Wiel, H. B. M., Jacobs, B., \& GazendamDonofrio, S. M. (2009). Burnout, engagement and resident physicians' self-reported errors. Psychology, Health \& Medicine, 14(6), 654-666.

Romppel, M., Braehler, E., Roth, M., \& Glaesmer, H. (2013). What is the general health questionnaire-12 assessing? Comprehensive Psychiatry, 54(4), 406-413.

Sanada, K., Montero-Marin, J., Alda Díez, M., Salas-Valero, M., PérezYus, M. C., Morillo, H., Demarzo, M., García-Toro, M., \& GarcíaCampayo, J. (2016). Effects of mindfulness-based interventions on salivary cortisol in healthy adults: a meta-analytical review. Frontiers in Physiology, 7(471), 1-12.

Sauvé, B., Koren, G., Walsh, G., Tokmakejian, S., \& Van Uum, S. H. (2007). Measurement of cortisol in human hair as a biomarker of systemic exposure. Clinical and Investigative Medicine, 30(5), 183191.

Shanafelt, T. D. (2009). Enhancing meaning in work: a prescription for preventing physician burnout and promoting patient-centered care. JAMA, 302(12), 1338-1340. 
Siegrist, J. (1996). Adverse health effects of high-effort/low-reward conditions. Journal of Occupational Health Psychology, 1(1), 27-41.

Siegrist, J., Starke, D., Chandola, T., Godin, I., Marmot, M., Niedhammer, I., \& Peter, R. (2004). The measurement of effortreward imbalance at work: European comparisons. Social Science \& Medicine, 58(8), 1483-1499.

Siegrist, J., Wege, N., Pühlhofer, F., \& Wahrendorf, M. (2009). A short generic measure of work stress in the era of globalization: effortreward imbalance. International Archives of Occupational and Environmental Health, 82(8), 1005-1013.

Spreitzer, G., Sutcliffe, K., Dutton, J., Sonenshein, S., \& Grant, A. (2005). A socially embedded model of thriving at work. Organization Science, 16(5), 537-549.

Stalder, T., \& Kirschbaum, C. (2012). Analysis of cortisol in hair - state of the art and future directions. Brain, Behavior, and Immunity, 26(7), 1019-1029.

Staufenbiel, S. M., Penninx, B. W. J. H., Spijker, A. T., Elzinga, B. M., \& van Rossum, E. F. C. (2013). Hair cortisol, stress exposure, and mental health in humans: a systematic review. Psychoneuroendocrinology, 38(8), 1220-1235.

Tang, Y.-Y., Hölzel, B. K., \& Posner, M. I. (2015). The neuroscience of mindfulness meditation. Nature Reviews Neuroscience, 16(4), 213 225.

Tyssen, R., Hem, E., Gude, T., Grønvold, N. T., Ekeberg, Ø., \& Vaglum, P. (2009). Lower life satisfaction in physicians compared with a general population sample: a 10 -year longitudinal, nationwide study of course and predictors. Social Psychiatry and Psychiatric Epidemiology, 44(1), 47-54.

van der Heijden, F., Dillingh, G., Bakker, A., \& Prins, J. (2008). Suicidal thoughts among medical residents with burnout. Archives of Suicide Research, 12(4), 344-346.
Verweij, H., Waumans, R. C., Smeijers, D., Lucassen, P. L., Donders, A. R. T., van der Horst, H. E., \& Speckens, A. E. (2016). Mindfulnessbased stress reduction for GPs: results of a controlled mixed methods pilot study in Dutch primary care. British Journal of General Practice, 66(643), e99-e105. https://doi.org/10.3399/ bjgp16X683497.

Verweij, H., van Ravesteijn, H., Lagro-Janssen, A. L. M., \& Speckens, A. E. M. (2018). Mindfulness-based stress reduction for residents: a randomized controlled trial. Journal of General Internal Medicine, $33(4), 429-436$.

Walach, H., Buchheld, N., Buttenmüller, V., Kleinknecht, N., \& Schmidt, S. (2006). Measuring mindfulness - the Freiburg Mindfulness Inventory (FMI). Personality and Individual Differences, 40(8), $1543-1555$.

Walach, H., Schmidt, S., \& Esch, T. (2014). Meditation intervention reviews. JAMA Internal Medicine, 174(7), 1193-1194.

Wallace, J. E., Lemaire, J. B., \& Ghali, W. A. (2009). Physician wellness: a missing quality indicator. The Lancet, 374(9702), 1714-1721.

Wittmann, M., \& Schmidt, S. (2014). Mindfulness meditation and the experience of time. In S. Schmidt \& H. Walach (Eds.), Meditation - neuroscientific approaches and philosophical implications (2nd ed., pp. 199-209). Heidelberg: Springer.

Wittmann, M., Rudolph, T., Linares Gutierrez, D., \& Winkler, I. (2015). Time perspective and emotion regulation as predictors of age-related subjective passage of time. International Journal of Environmental Research and Public Health, 12(12), 16027-16042.

Publisher's Note Springer Nature remains neutral with regard to jurisdictional claims in published maps and institutional affiliations. 